\section{EUROPÄISCHE IMPFWOCHE}

\section{Ziel der Masernelimination fest im Visier}

Die Woche vom 24. April bis 1. Mai steht im Zeichen der Schutzimpfung. Ziel der „,5. Europäischen Impfwoche“, einer WHO-Initiative, ist es, europaweit Ärzte und Patienten für die Bedeutung von Impfmaß nahmen zu sensibilisieren und mit Plakataktionen, Fortbildungsveranstaltungen und Impfaktionen

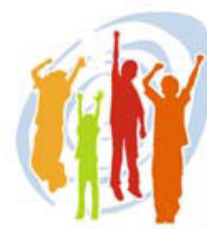

Vorbeugen Schützen Impfen

für bestimmte Zielgruppen den Impfschutz der EU-Bevölkerung zu verbessern. Ein besonderer Schwerpunkt liegt auf dem bereits vor zehn Jahren vereinbarten Ziel der Masernelimination, das bis Ende 2010 erreicht sein soll. Weitere Informationen unter: Europäische Impfwoche Epidemiologisches Bulletin www.euro.who.int/eiw 10/2010

\section{MÄNNLICHE HORMONPROBLEME}

\section{Niedriger Testosteronspiegel $=$ früher Tod?}

Männer mit niedrigem Testosteronspiegel sterben früher. Diesen Zusammenhang haben Wissenschaftler der Universitäten Greifswald und Erlangen-Nürnberg nachgewiesen. Auf der Basis der seit zehn Jahren laufenden Greifswalder Bevölkerungsstudie (SHIP) wurden rund 2000 Männer zwischen 20 und 79 Jahren analysiert. Bei $15-20 \%$ der Probanden über 50 wurde ein erniedrigter Testosteronspiegel (unter 8,7 nmol/l) festgestellt. Diese Männer hatten ein mehr als doppelt so hohes Mortalitätsrisiko wie Probanden mit normalen Hormonwerten. Bei Männern mit vermindertem Testosteronspiegel bestanden zudem oft weitere gesundheitliche Probleme wie Fettleibigkeit, Fettstoffwechselstörungen und Fettleber. Auch Hypertonie und Diabetes traten gehäuft auf. Eur Heart J 2010; doi:10.1093/eurheartj/ehqoog

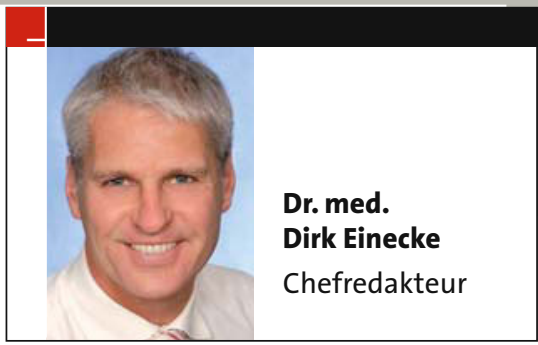

\section{ALZHEIMER-PRÄVENTION}

\section{Essen gegen das Vergessen}

Wer reichlich Nüsse, Fisch, Tomaten, Geflügel, Gemüse und Obst zu sich nimmt und fette Milchprodukte, Butter und rotes Fleisch meidet, könnte dadurch sein Alzheimerrisiko senken. Ärzte der ColumbiaUniversität propagieren diese „protektive Diät" auf der Grundlage einer Studie mit 2148 älteren Patienten, von denen 253 im Lauf von knapp vier Jahren an einer Alzheimerdemenz erkrankten. Teilnehmer, die sich weitgehend mit der beschriebenen mediterranen Diät ernährten, hatten ein deutlich geringeres Risiko als diejenigen, die fett- und fleischreich aßen. Arch Neurol 2010;67(6)

\section{ADIPOSITASRISIKEN}

\section{Cotrimoxazol erhöht Blutungsgefahr}

Bei Patienten unter Vitamin-K-Antagonisten sollten Harnwegsinfekte möglichst nicht mit Cotrimoxazol behandelt werden, raten kanadische Ärzte. Grundlage für ihre Empfehlung sind Daten von 134637 Warfarinpatienten, von denen 2151 wegen einer Blutung im oberen Gastrointestinaltrakt stationär behandelt wurden. Das Ri- siko für diese Komplikation war unter Cotrimoxazol vierfach höher. Ein erhöhtes Risiko fand sich auch unter Ciprofloxacin (RR 1,9), aber nicht unter anderen Antibiotika. Cotrimoxazol hemmt das an der Metabolisierung von Warfarin und Phenprocoumon beteiligte Cytochrom P450 2C9. Arch Intern Med 2010;170:617-621

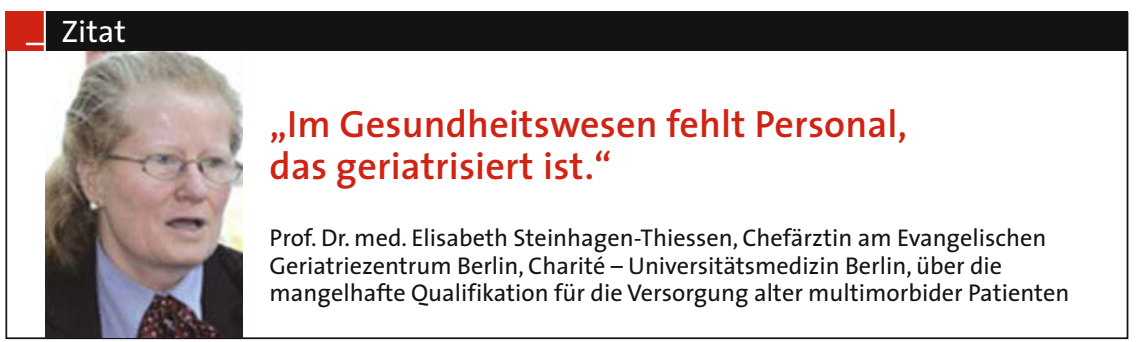

\section{Dicke Mutter - herzkrankes Kind}

Je adipöser die Mutter, desto größer ist ihr Risiko, ein Kind mit Herzfehler auf die Welt zu bringen. Frauen mit einem BMI über 30 haben bereits ein um $15 \%$ erhöhtes Risiko, $\mathrm{ab}$ einem BMI über 40 ist das Risiko gegenüber normalgewichtigen Frauen sogar verdoppelt. Leichtes Übergewicht oder Untergewicht geht hingegen nicht mit einem signifikant veränderten kardialen Missbildungsrisiko einher. Dieses Ergebnis einer bevölkerungsbasierten Fall-KontrollStudie in New York liefert ein weiteres Argument dafür, bei Schwangeren auf ein möglichst normales Gewicht zu achten. Am J Clin Nutr 2010;DOI:10.3945/ajcn.2009.28865 